\title{
A foodborne outbreak of Salmonella Bareilly in the United Kingdom, 2010
}

P Cleary (paul.cleary@hpa.org.uk)1, L Browning ${ }^{2}$, J Coia ${ }^{3}$, J Cowden², A Fox ${ }^{4}$, J Kearney5, C Lane ${ }^{6}$, H Mather³, C Quigley¹, Q Syed ${ }^{1}$, D Tubin-Delic ${ }^{7}$, on behalf of the outbreak control team

1. Health Protection Agency North West region (HPA NW), Liverpool, United Kingdom

2. Health Protection Scotland (HPS), Glasgow, United Kingdom

3. Scottish Salmonella, Shigella and Clostridium difficile Reference Laboratory, Glasgow, United Kingdom

4. Health Protection Agency Food Water and Environmental Microbiology Laboratory, Preston, United Kingdom

5. Health Protection Agency East of England region, Witham, Essex, United Kingdom

6. Health Protection Agency Gastrointestinal, Emerging and Zoonotic Infections Department (GEZI) - Epidemiological Services, London, United Kingdom

7. Food Standards Agency, London, United Kingdom

Citation style for this article:

Cleary P, Browning L, Coia J, Cowden J, Fox A, Kearney J, Lane C, Mather H, Quigley C, Syed Q, Tubin-Delic D, on behalf of the outbreak control team. A foodborne outbreak of Salmonella Bareilly in the United Kingdom, 2010. Euro Surveill. 2010;15(48):pii=19732. Available online: http://www.eurosurveillance.org/ViewArticle. aspx?Articleld $=19732$

Article published on 2 December 2010

We report the preliminary findings of the investigation of an outbreak of foodborne Salmonella Bareilly. Between August and November 2010, there were 231 laboratory-confirmed reports of $S$. Bareilly in the United Kingdom. A case-control study showed that consumption of bean sprouts was significantly associated with illness. The investigation concluded that raising public awareness to ensure the correct preparation of raw bean sprouts during cooking was the principal means of preventing further cases.

\section{Background}

Consumption of bean sprouts has previously been associated with outbreaks of Salmonella infection $[1,2]$. There was previously an outbreak of S. Saint-Paul infection associated with bean sprouts in the United Kingdom (UK) in 1988 [3].

Salmonella enterica subsp. enterica serovar Bareilly, or $S$. Bareilly, is a group $C_{1}$ serovar first identified in India in 1928 [4]. In years when no outbreaks occur, on average 30 to 50 cases are reported in England and two to five cases in Scotland.

The current investigation began on 27 August 2010, when an outbreak of S. Bareilly was reported following a wedding reception in Greater Manchester in northwest England. An increase in the number of detected cases of S. Bareilly was also reported in Scotland on 2 September 2010 and more widely in England on 10 September 2010. The outbreak was first reported to the European Centre for Disease Prevention and Control (ECDC) Epidemic Intelligence Information System (EPIS) site on 14 September, providing an image of the outbreak pulsed-field gel electrophoresis (PFGE) profile. Between 14 September and 1 October, 10 EPIS members responded with no indication of co-temporal increases in findings of this serovar. One country (Ireland) reported the same profile in a case who had visited London in September, but no further exposure data was available.

Preliminary information from cases in Scotland, from the wedding outbreak investigation, and from trawling questionnaires including questions on a comprehensive range of possible risk factors including foodstuffs, raised the hypothesis of salad leaves and/or bean sprouts as possible vehicles of infection. A matched case-control study including 34 cases meeting the case definition was conducted across the UK beginning on 21 September 2010. At the time the case-control study started there were some 40 cases who had not done the trawling questionnaire and who were not linked to the wedding outbreak.

Between the beginning of August and the 19 November 2010, there were 231 laboratory-confirmed reports of S. Bareilly across the UK. 10 of these were linked to the wedding in Greater Manchester.

\section{Case-control study}

Definition of cases and controls

The case-control study was conducted using telephone interviews with cases of $S$. Bareilly fitting the confirmed case definition and with controls selected by sequential digit dialling based on the telephone number of the case, to match on broad geographical location of residence.

Confirmed cases were defined as persons aged 18 and over with microbiologically confirmed infection with S. Bareilly with a sample received at the Health Protection Agency (HPA) Salmonella Reference Unit or the Scottish Salmonella, Shigella and Clostridium difficile Reference Laboratory on or after 1 August 2010 with (i) no history of foreign travel or close contact with a case of diarrhoea in the seven days prior to the onset of illness, (ii) who did not attend the wedding in the 
northwest of England associated with the point source outbreak and (iii) who had not been previously interviewed as part of the preliminary investigation.

Controls were defined as residents of England, Wales, Northern Ireland or Scotland aged 18 and over residing in the area covered by the same telephone exchange as their matched cases who had not had gastrointestinal symptoms (vomiting and/or diarrhoea) in the seven days prior to interview, and who had no history of foreign travel or of close contact with a case of diarrhoea in the seven days prior to interview. The person answering the telephone was recruited unless they were a child, in which case interviewers asked to speak to an adult. The case-control ratio was 1:2.

\section{Interviews}

Data were collected on demographics, clinical features and outcomes, travel history, infectious contacts, and consumption of salad leaves, bean sprouts or alfalfa sprouts in the three days prior to illness (or three days prior to interview for controls). Information on travel history and infectious contacts was collected to check eligibility. After descriptive and univariate analysis, forward stepwise conditional logistic regression was conducted using the statistical software R [5]. Possible risk factors with a $\mathrm{p}$ value of $\mathbf{0 . 2}$ in univariate analysis were added sequentially after assessment for multicollinearity.

\section{Laboratory investigation of food items}

Samples of bean sprouts (100 g) were collected from suppliers B and C. They were examined using HPA standard methods, based on BS EN ISO 6579:2002, for the detection of Salmonella spp, modified to include extended incubation for 48 hours for both enrichment media (Muller-Kauffmann tetrathionate novobiocin broth and Rappaport-Vassiliadis Soya Peptone broth) and selective solid media Xylose Lysine Desoxycholate and Brilliant Green agars [6].

\section{FIGURE}

Epidemic curve of $S$. Bareilly cases in England, Wales and Northern Ireland ${ }^{\mathrm{a}}, 2010(\mathrm{n}=213)$

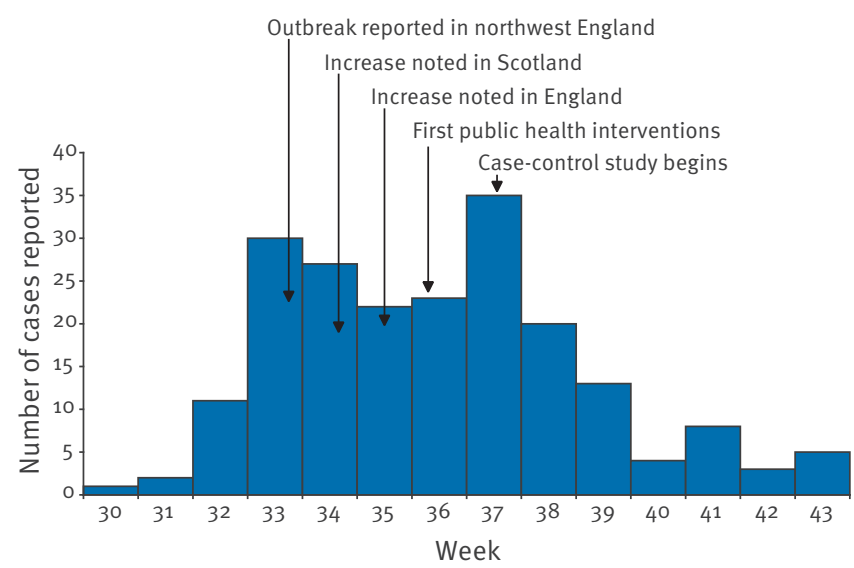

a By specimen date.

\section{Results of case-control study}

Data on 34 cases (response rate of $77 \%$ ) meeting the case definition, with onset dates ranging from 13 September 2010 to 14 October 2010, and 64 eligible controls were collected. Cases had a mean age of 49 years (standard deviation 15.1 years) and 21 of 33 cases were female. A total of 32 of 34 reported diarrhoea and seven of 34 reported vomiting. Five cases had been hospitalised as a result of their infection. Cases reported a median duration of illness of 7.5 days (range 2 to 30 days).

In univariate analysis, consumption of bean sprouts, consumption of any salad leaves and age younger than 65 years were all significantly associated with illness. In the final conditional logistic regression model, only consumption of bean sprouts was significantly associated with illness (crude matched odds ratio (OR) 8.3, $95 \%$ confidence interval $(\mathrm{Cl}) 1.8$ to 38.7 ; adjusted matched OR $6.8,95 \% \mathrm{Cl} 1.4$ to 33.0 ). Of the 15 cases who recalled consumption of bean sprouts, six had eaten them in the home (four had purchased them from supermarkets). 10 had eaten them in take away meals or at restaurants. (One person had eaten them from both sources.)

In total, 94 of the 231 patient isolates underwent further typing and 87 of these were found to be of PFGE type SBARXB.0016.

\section{Food investigation}

The complex distribution network of bean sprouts and the results of routine microbiological testing of bean sprouts by suppliers for quality control purposes in the UK were also investigated. In August and September 2010, two UK suppliers identified in the investigation of the Scottish cases (suppliers A and B) had recorded the identification of a group C Salmonella in samples of bean sprouts which were intended to be cooked by consumers. None of these isolates were available for further characterisation. Routine regular testing of samples at an upstream supplier (supplier C, who supplies $B$, who in turn supplies $A$ ) had been consistently negative for many years.

Samples of bean sprouts from suppliers B and $C$ were collected. S. Bareilly of a PFGE type indistinguishable from the outbreak cases was identified in a packet of bean sprouts produced by supplier C. Supplier C received mung bean seeds from upstream suppliers who sourced mung bean seeds from China or Myanmar. The investigation is ongoing.

\section{Discussion}

Our epidemiological and microbiological investigations implicated bean sprouts as a vehicle for $S$. Bareilly transmission, consistent with previous research showing that bean sprouts can be a vehicle for Salmonella transmission [1].

Case-control study designs are prone to a number of biases, the most important being recall bias. Although 
there was a small amount of press interest in the possible link between $S$. Bareilly and bean sprouts at the time of the case-control study it is unlikely that many people in the general public were aware of a possible link between Salmonella and bean sprouts. In our study, controls were selected by random digit dialling, which may result in a low response rate and controls that are not representative of the general population. This is however unlikely to account for the strong observed association in our study.

Bean sprouts follow a complex path from farm to table that includes growing, harvesting, processing and shipping of mung bean seeds, followed by sprouting (normally at temperatures of $20-30{ }^{\circ} \mathrm{C}$ with high humidity) and distribution of the finished product. Seeds may arrive already contaminated or contamination may occur at any point of production and distribution. As in previous outbreaks, this investigation concluded that the seeds were likely to have been contaminated, as investigations at suppliers found little potential for cross contamination of sprouted seeds [7]. Based on the experience of this investigation, the methodology used for routine microbiological quality control testing of bean sprouts may not be sensitive to low levels of Salmonella contamination. This may have implications for future testing protocols.

The bean sprouts implicated in this investigation were not ready to eat products and would be safe to eat if the instructions for correct preparation (washing and cooking until piping hot) were followed. Public health interventions resulting from this investigation focussed on communications to the public and to public and environmental health professionals advising of the correct preparation of bean sprouts, and on improving food labelling where this was ambiguous. Given that $S$. Bareilly was only detected in raw mung bean sprouts intended to be cooked, rather than ready to eat bean sprouts, and the producers and suppliers have not been found to be at fault in our investigations, no product recalls were deemed necessary. No other interventions besides addressing the issue of potentially misleading labels were put in place with suppliers and producers. At the time of writing, the numbers of reports of $S$. Bareilly infection in the UK had fallen back to near expected levels.

References

1. Taormina PJ. Beuchat LR \& Slutsker L Infections associated with eating seed sprouts: an international concern. Emerg Infect Dis. 1999;5(5):626-34.

2. Outbreak of salmonellosis in a restaurant in Stockholm, Sweden, September - October 2006. Euro Surveill. 2007;12(11):pii=749. Available from: http://www. eurosurveillance.org/ViewArticle.aspx?Articleld=749

3. O'Mahony M, Cowden J, Smyth B, Lynch D, Hall M, Rowe B, et al. An outbreak of Salmonella Saint-Paul infection associated with beansprouts. Epidemiol Infect. 1990;104(2):229-35.

4. Bridges RF, Scott WM. A new organism causing paratyphoid fever in India. J Roy Army Med Corps. 1931;56:241-9.

5. The R Development Core Team. R: A Language and Environment for Statistical Computing. Vienna:2010. Available from: http:// www.R-project.org
6. British Standards Institution (BSI). Microbiology of food and animal feeding stuffs-horizontal method for detection of Salmonella spp. London:BSI;2002.

7. Mohle-Boetani JC, Farrar J, Bradley P, Barak JD, Miller M, Mandrell R, et al. Salmonella infections associated with mung bean sprouts: epidemiological and environmental investigations. Epidemiol Infect. 2009;137(3):357-66. 